\title{
Mechanisms underlying the antireflux action of fundoplication
}

\author{
A C Ireland, R H Holloway, J Toouli, J Dent
}

\begin{abstract}
The effect of fundoplication on patterns of gastrooesophageal reflux and the underlying motor mechanisms were investigated in 18 patients with symptomatic reflux. Oesophageal motility and $\mathrm{pH}$ were recorded concurrently after a standard meal. Studies were performed preoperativey and from 5 to 27 months after surgery. Fundoplication virtually eliminated reflux in all but three patients. Control of reflux was associated with a $\mathbf{5 0 \%}$ fall in the number of transient lower oesophageal sphincter relaxations, a fall in the proportion of transient lower oesophageal sphincter relaxations accompanied by reflux from $47 \%$ to $17 \%$, and an increase in the mean residual pressure at the gastro-oesophageal junction during swallow induced lower oesophageal sphincter relaxation from $0.7 \mathrm{~mm} \mathrm{Hg}$ to $6.0 \mathrm{~mm} \mathrm{Hg}$. Basal pressure at the gastro-oesophageal junction rose from $10.9 \mathrm{~mm} \mathrm{Hg}$ to $14.5 \mathrm{~mm} \mathrm{Hg}$, however, there was no correlation between postoperative reflux and basal gastro-oesophageal junction pressure. These findings suggest that the antireflux effects of fundoplication result from changes in the mechanical behaviour of the gastro-oesophageal junction that result in incomplete abolition of the high pressure zone during lower oesophageal sphincter relaxation, and reduced triggering of transient lower oesophageal sphincter relaxations.

(Gut 1993; 34: 303-308)
\end{abstract}

Rudolph Nissen discovered serendipitously that fundoplication prevents reflux when he reviewed a patient 16 years after partial oesophagectomy.' The oesophago-gastric anastomosis in this patient had been wrapped by the fundus of the stomach, not with the aim of preventing reflux, but rather to protect against anastomotic leakage. Fundoplication has subsequently

Summary of clinical details of patients

\begin{tabular}{lllllll}
\hline $\begin{array}{l}\text { Patient } \\
\text { no }\end{array}$ & Sex & $\begin{array}{l}\text { Age } \\
(y r)\end{array}$ & $\begin{array}{c}\text { Time after } \\
\text { surgery }(\text { mth })\end{array}$ & $\begin{array}{l}\text { Endoscopy } \\
\text { findings }\end{array}$ & Histology & Bernstein \\
\hline test
\end{tabular}

$\mathrm{ND}=$ not done $;$ Pos $=$ positive . become the most widely used form of antireflux surgery, the efficacy of which has been established by clinical and endoscopic follow up, and by oesophageal $\mathrm{pH}$ monitoring. ${ }^{2-5}$ Nevertheless, surgical methods of controlling reflux have developed largely on an empirical basis, without a well defined concept of how they work.

Recent manometric studies performed on patients after fundoplication have documented an increased pressure at the gastro-oesophageal junction which has been attributed to an increase in basal lower oesophageal sphincter tone. It is not clear by which mechanism fundoplication increases the pressure at the gastro-oesophageal junction, however, and this change in gastrooesophageal junction pressure does not correlate with successful control of reflux. ${ }^{67}$

In most patients with reflux disease, reflux has been shown to occur mainly during transient lower oesophageal sphincter relaxations that are distinct from swallow induced lower oesophageal sphincter relaxations. ${ }^{89}$ The effect of fundoplication on the incidence and patterns of transient sphincter relaxations has not been reported. Swallow induced relaxation of the gastrooesophageal junction has been found to be incomplete in patients with fundoplication. ${ }^{71011}$ It has been suggested that this incomplete relaxation is relevant to the prevention of reflux. None of the studies, however, has included analysis of the spontaneous patterns of lower oesophageal sphincter function and mechanisms of reflux before and after fundoplication. In this study we examined the effect of fundoplication on spontaneous patterns of reflux and the motor mechanisms underlying the reflux episodes.

\section{Methods}

\section{PATIENTS}

We studied 18 patients, recruited between 1979 and 1981, with well documented symptomatic gastro-oesophageal reflux that was refractory to medical treatment. Symptomatic reflux was defined as erosive or ulcerative oesophagitis, and/or symptoms that were clearly related to oesophageal acidification during either spontaneous reflux, that was witnessed during concurrent postprandial oesophageal manometry and $\mathrm{pH}$ monitoring, or during a Bernstein test. Patients were judged to be refractory to medical management if they continued to complain of disabling symptoms or had persistent erosive or ulcerative oesophagitis despite aggressive medical treatment including cimetidine. The clinical details are summarised in the Table. Studies were performed before and from 4 to 28 months after fundoplication with an identical protocol. A similar surgical technique was used by the three senior surgeons who contributed to 
the study. Fundoplication was performed transabdominally using a complete wrap over a 52 French gauge bougie.

\section{STUDY PROTOCOL}

The study protocol was approved by the Ethical Review Committee of the Flinders Medical Centre. Patients fasted for at least 4 hours and refrained from smoking for a minimum of 6 hours before the study. Anticholinergic and antisecretory drugs were stopped 12 hours before the study. During the study the patients remained recumbent, either supine or lying on their side. Oesophageal manometry and $\mathrm{pH}$ were recorded concurrently during a 1 hour fasting period and for 4 hours after a standard meal consisting of soft meat or fish, mashed potatoes, cooked vegetables, ice cream and $180 \mathrm{ml}$ of milk $(2595 \mathrm{~kJ})$. Patients were not allowed to doze, because of the effect of sleep on oesophageal motility and reflux patterns. ${ }^{8}$ After the monitoring period, relaxation of the gastro-oesophageal junction was assessed in response to swallowing of $10,5 \mathrm{ml}$ water boluses.

MANOMETRIC AND PH MONITORING TECHNIQUES Oesophageal motility was monitored continuously using an eight lumen manometric assembly that incorporated a sleeve sensor. ${ }^{12}$ The sleeve was positioned so that it straddled the gastro-oesophageal high pressure zone. Two side holes, 1 and $3 \mathrm{~cm}$ below the distal margin of the sleeve, recorded intragastric pressure. Side holes at $2,7,14$, and $21 \mathrm{~cm}$ proximal to the upper sleeve margin monitored motor activity of the oesophageal body. A side hole located in the pharynx monitored swallows. Each lumen was connected in-series with a pressure transducer and perfused with distilled water by a low compliance pneumohydraulic pump. The sleeve and gastric manometric channels were perfused at a rate of $0.5 \mathrm{ml} / \mathrm{minute}$. The oesophageal body and pharyngeal channels were perfused at $0.13 \mathrm{ml} /$ minute, a rate selected to yield reliable recognition of the occurrence of oesophageal body and pharyngeal contractions, yet to minimise changes in the pattern of oesophageal acid clearance or the rate of primary or secondary peristalsis.

Oesophageal $\mathrm{pH}$ was monitored with either a Beckman (no 39042) or a Microelectrodes (no MI-506) unipolar intraluminal $\mathrm{pH}$ electrode positioned $5 \mathrm{~cm}$ above the proximal margin of the gastro-oesophageal junction. The electrode was calibrated in buffers of $\mathrm{pH} 4$ and 7 before and after each study. An electrode drift in excess of $0.6 \mathrm{pH}$ units led to rejection of any study from the analysis. Correction for electrode drifts of $<0.5 \mathrm{pH}$ units was made on the assumption that the drift was linear during the study. Signals from the pressure transducers and $\mathrm{pH}$ meter (Electronic Instruments Ltd, no 2320) were processed and recorded on a Devices M19 chart recorder at a paper speed of $80 \mathrm{~mm} /$ minute.

DATA ANALYSIS

Only the 4 hour postprandial period was analysed. The oesophageal $\mathrm{pH}$ tracing was scored manually to determine the percentage of time the oesophagus was exposed to a $\mathrm{pH}<4$. Reflux episodes were also identified and counted, being defined as either a drop of oesophageal $\mathrm{pH}$ below 4 for at least 4 seconds or, if oesophageal $\mathrm{pH}$ was already below 4 , as a decrease of at least $1 \mathrm{pH}$ unit sustained for at least 4 seconds.

Basal end-expiratory pressure at the gastrooesophageal junction was determined at 15 minute intervals by taking a 1 minute visual mean of the tracing. Separate mean values were obtained for each patient. The completeness of relaxation of the gastro-oesophageal junction was evaluated at the end of the monitoring period by determining the difference between intragastric pressure and the nadir of gastrooesophageal junction pressure during water swallow induced gastro-oesophageal junction relaxations.

The tracings were also analysed for transient lower oesophageal sphincter relaxations with the $\mathrm{pH}$ tracing masked. For the preoperative studies, transient lower oesophageal sphincter relaxations were defined as a sudden drop of pressure at the gastro-oesophageal junction of $\geq 5 \mathrm{~mm} \mathrm{Hg}$ to within $2 \mathrm{~mm} \mathrm{Hg}$ of intragastric pressure, not preceded by a pharyngeal swallow signal within the previous 5 seconds, and lasting longer than 5 seconds. For the postoperative studies, transient lower oesophageal sphincter relaxations were scored if the nadir pressure during relaxation was on or below the residual relaxation pressure determined during the water swallows. Separate note was made of those transient relaxations whose nadir pressures reached $\leq 2 \mathrm{~mm} \mathrm{Hg}$ relative to intragastric pressure during the postoperative studies. The manometric tracings were also analysed for the pattern of pharyngeal and oesophageal motor function just before, and at the time of the onset of acid reflux using criteria applied previously.

Statistical analyses of the rate of reflux episodes and the duration of oesophageal acid pressure were performed using Wilcoxon's signed rank test and the Mann-Whitney $U$ test. Basal gastro-oesophageal junction pressure measurements were analysed using analyses of variance, and swallow induced nadir pressures were compared using the paired t-test.

\section{Results}

GASTRO-OESOPHAGEAL REFLUX

Fundoplication substantially reduced postprandial oesophageal acid exposure from a mean of $19.9(5.9) \%$ to $2.3(1.2) \%(\mathrm{p}<0.001$, Fig 1$)$. Fundoplication also significantly reduced the number of reflux episodes from a total of 327 (mean $18 \cdot 2(4 \cdot 3)$ ) before surgery to 53 (mean 2.9 $(1 \cdot 6))$ after surgery $(\mathrm{p}<0 \cdot 001)$. Reflux episodes occurred in only six of the 18 patients after surgery, and in only three was the distal oesophageal $\mathrm{pH}$ below 4 for more than $2 \%$ of the postprandial time (upper limit of normal $<4 \%{ }^{9}$ )

Analysis of the motor events associated with the onset of reflux episodes showed that, before fundoplication, transient lower oesophageal 
Figure 1: Duration of postprandial oesophageal acid exposure before and after fundoplication. Each point represents data for an individual patient.

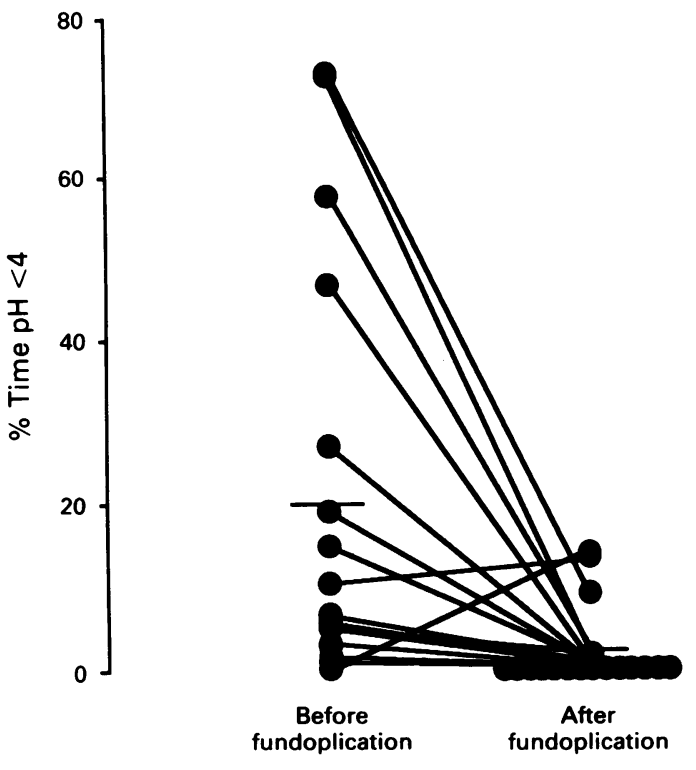

sphincter relaxation was the most prevalent mechanism of reflux in 16 of the 18 patients. Although swallow induced lower oesophageal sphincter relaxation was the most prevalent mechanism overall, 92 of the 106 reflux episodes that occurred by this mechanism, occurred in only two patients. The distribution of the patterns of lower oesophageal sphincter function associated with reflux after surgery was similar to that preoperatively (Fig 2).

LOWER OESOPHAGEAL SPHINCTER RELAXATION The mean nadir pressure at the gastrooesophageal junction at maximum swallow induced lower oesophageal sphincter relaxation preoperatively was $0.7(0.5) \mathrm{mm} \mathrm{Hg}$, and rose to 6.0 (4.2) $\mathrm{mm} \mathrm{Hg}$ after surgery (Fig 3 ).

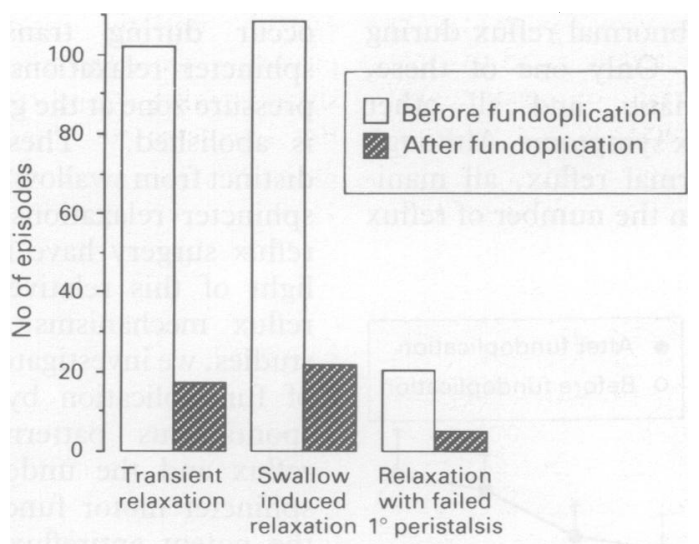

Figure 2: Patterns of lower oesophageal sphincter (LOS) function during reflux episodes. The bars indicate the total number of reflux episodes occurring with each type of LOS motor pattern. Swallow induced relaxation - relaxation associated with normal primary peristalsis failed $I^{0}$ peristalsis swallow induced LOS relaxation associated with failed peristalsis; absent

basal pressure - absent basal LOS pressure for at least 30 seconds before reflux; stable detectable pressure - reflux occuring during a period of stable detectable sphincter pressure without evidence of straining; strain - reflux occurring because of increased intra-abdominal pressure during physical straining sufficient to overcome basal LOS pressure; not interpretable pattern of LOS function obscured by movement, straining or rapid motor events.

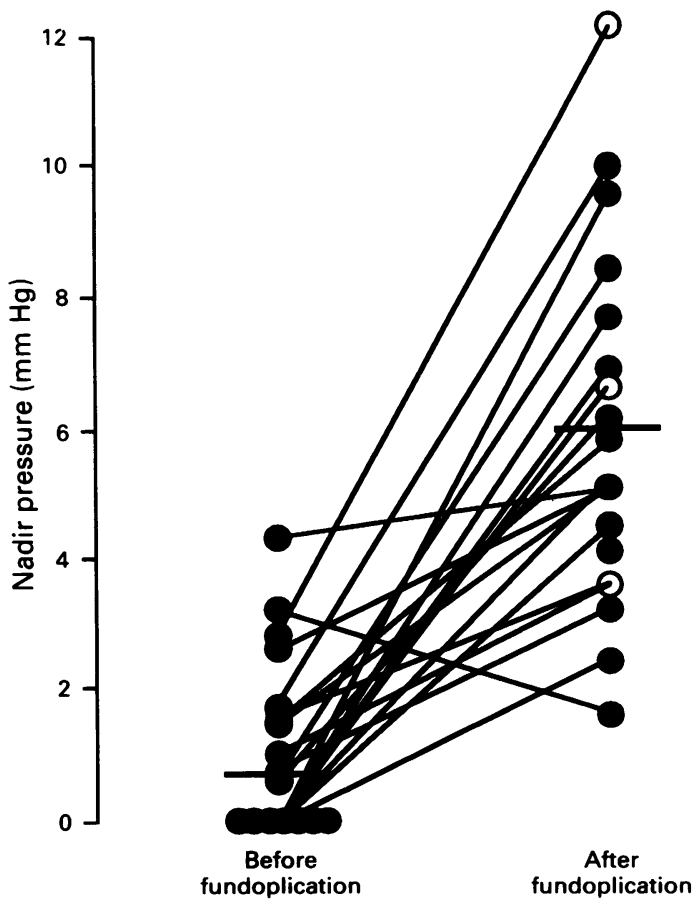

Figure 3: Residual pressure at the gastro-oesophageal junction during swallow induced lower oesophageal sphincter relaxation before and after fundoplication. The open circles denote patients with recurrent reflux.

\section{TRANSIENT LOWER OESOPHAGEAL SPHINCTER}

RELAXATIONS

Fundoplication was associated with a reduction in the total number of postprandial transient lower oesophageal sphincter relaxations from 197 to $100(p<0.01)$. Most of these were spontaneous, with no antecedent motor activity in the pharynx or oesophageal body (Fig 4). Fifty two of the 100 transient lower oesophageal sphincter relaxations that occurred postoperatively had residual gastro-oesophageal junction pressures $>2 \mathrm{~mm} \mathrm{Hg}$ (Fig 5). Thus, if the preoperative criterion of completeness of relaxation had been applied to the scoring of postoperative tracings, only 48 transient lower oesophageal sphincter relaxations would have been deemed to have occurred. A weak but significant direct relationship was found between the nadir lower oesophageal sphincter pressure during swallow induced sphincter relaxation and the proportion

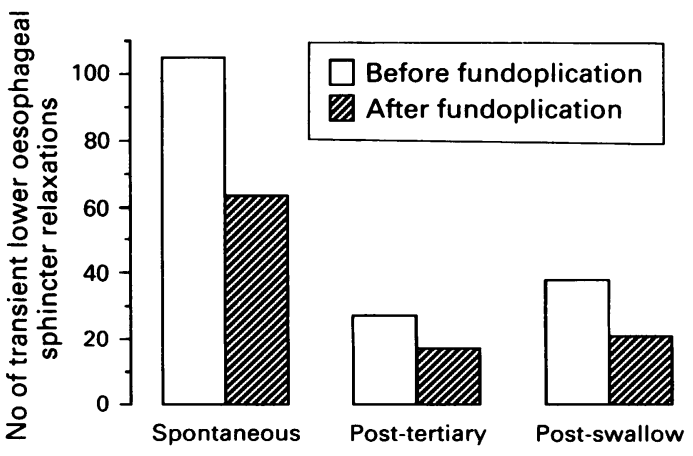

Figure 4: Patterns of transient lower oesophageal sphincter (LOS) relaxation before and after fundoplication. Each bar indicates the total number of relaxations of each type. Spontaneous - LOS relaxation without any antecedent motor activity in the oesophageal body; post-tertiary - LOS relaxation following synchronous contractions in the distal oesophagus, post-swallow - LOS relaxation immedaitely following the LOS contraction that follows a swallow induced LOS relaxation (ref 9 ). 


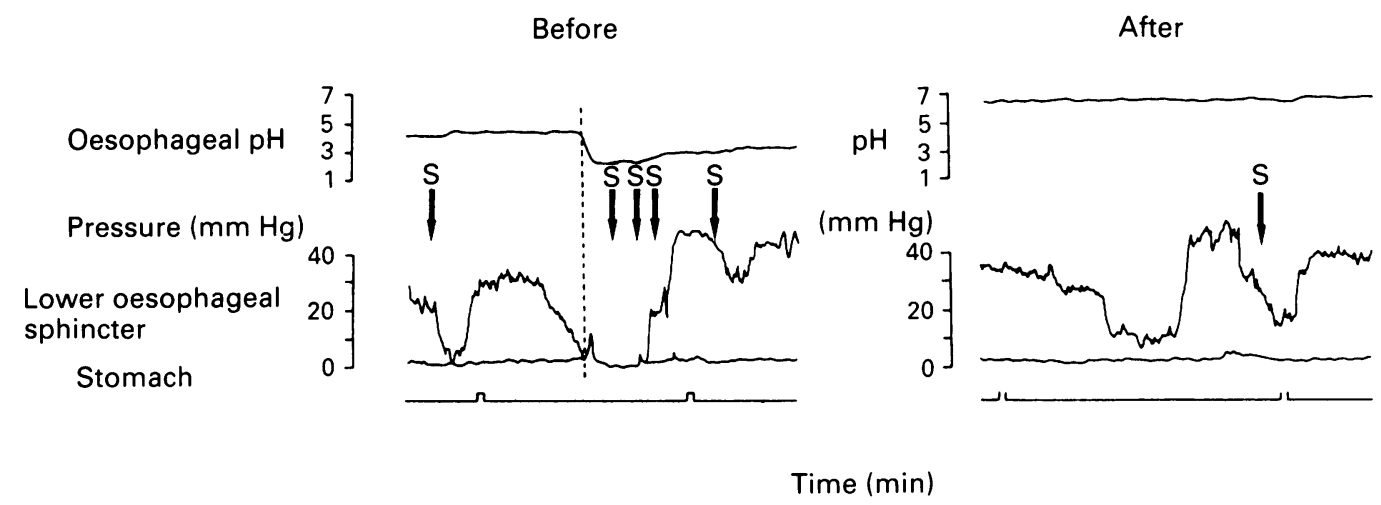

Figure 5: Tracings showing patterns of lower oesophageal sphincter (LOS) relaxation before and after fundoplication. The LOS and gastric tracings have been superimposed. Before fundoplication spontaneous swallows $(S)$ induce complete LOS relaxation. A prolonged relaxation associated with multiple swallows is associated with gastro-oesophageal reflux. After fundoplication, $L O S$ relaxation is incomplete, leaving a residual pressure of about $8 \mathrm{~mm} \mathrm{Hg}$ during both a transient LOS relaxation and a swallow induced relaxation. No reflux has occurred.

of incomplete transient relaxations $(r=0.58$, $\mathrm{p}<0.05)$. Preoperatively, gastro-oesophageal reflux occurred during $47 \%$ of transient lower oesophageal sphincter relaxations. Postoperatively, this value fell to $19 \%$ of relaxations with a residual pressure of $\leq 2 \mathrm{~mm} \mathrm{Hg}$, and $15 \%$ of relaxations with residual pressures of $>2 \mathrm{~mm} \mathrm{Hg}$.

BASAL GASTRO-OESOPHAGEAL JUNCTION PRESSURE The pattern of basal pressure at the gastrooesophageal junction, both pre- and postprandially and before and after surgery is illustrated in Figure 6. Basal pressure rose after surgery from an overall postprandial mean of $10.9(2.7) \mathrm{mm} \mathrm{Hg}$ to $14.5(1.5) \mathrm{mm} \mathrm{Hg}$ $(p<0.02)$. There was no correlation between either basal gastro-oesophageal junction pressure postoperatively, or the change in gastrooesophageal junction pressure and postoperative reflux.

\section{PATIENTS WITH RECURRENT REFLUX}

Three patients showed abnormal reflux during the postoperative study. Only one of these, however, was symptomatic, and all other patients were free of reflux symptoms. Although these patients had abnormal reflux, all manifested at least a $60 \%$ fall in the number of reflux

Figure 6: Basal lower oesophageal sphincter (LOS) pressure before and after fundoplication. Data are expressed as mean (SEM) for each hour of the recording period.

Fundoplication was associated with a significant rise in LOS pressure $(p<0 \cdot 02)$. episodes postoperatively. This fall was the result of a comparable fall in the number of reflux episodes occurring during transient and swallow induced lower oesophageal sphincter relaxations. Although a $50 \%$ fall occurred in the overall number of transient sphincter relaxations, in contrast to the operative successes, the proportion of transient relaxations accompanied by reflux did not change after surgery. While most (28 out of 43) of the postoperative reflux episodes occurred in one patient, the distribution of patterns of lower oesophageal sphincter function associated with reflux postoperatively was similar to that preoepratively. Basal lower oesophageal sphincter pressure and nadir pressure during swallow induced lower oesophageal sphincter relaxation (Fig 3) were similar to those in patients without recurrent reflux.

\section{Discussion}

Over the past decade, combined manometric and $\mathrm{pH}$ recordings have shown that at rest virtually all reflux episodes in healthy subjects, and most reflux episodes in patients with reflux disease, occur during transient lower oesophageal sphincter relaxations, during which the high pressure zone at the gastro-oesophageal junction is abolished. ${ }^{89}$ These relaxations are clearly distinct from swallow induced lower oesophageal sphincter relaxations. The mechanics of antireflux surgery have not been evaluated in the light of this relatively new understanding of reflux mechanisms. In contrast to previous studies, we investigated the mechanism of action of fundoplication by examining its effect on spontaneous patterns of gastro-oesophageal reflux and the underlying lower oesophageal sphincter motor function. Our study confirms the potent antireflux effect of fundoplication, and shows further that this effect is associated with a reduction in the rate of transient lower oesophageal sphincter relaxations as well as the proportion of transient lower oesophageal sphincter relaxations accompanied by reflux, and an increase in the residual pressure at the gastro-oesophageal junction during sphincter relaxation.

A major effect of fundoplication was the substantial $50 \%$ reduction in the number of transient lower oesophageal sphincter relaxa- 
tions. This fall was evident despite a more liberal definition of transient lower oesophageal sphincter relaxations postoperatively. The estimates of the numbers of transient lower oesophageal sphincter relaxations were difficult to make postoperatively because our standard definition includes the stipulation that sphincter pressure drops to $2 \mathrm{~mm} \mathrm{Hg}$ or less, relative to intragastric pressure. ${ }^{13}$ In the postoperative studies, there were 52 abrupt decreases in gastrooesophageal junction pressure, that did not reach a nadir within $2 \mathrm{~mm} \mathrm{Hg}$ of intragastric pressure, which satisfied criteria and so were scored as transient lower oesophageal sphincter relaxations. These relaxations had the distinctive temporal profile and patterns of associated oesophageal motility of transient lower oesophageal sphincter relaxations before fundoplication. Because the data from the present study and others indicate that fundoplication limits swallow induced lower oesophageal sphincter relaxation during water swallows by what seems to be a purely mechanical effect that is extrinsic to the sphincter, we modified the definition of transient lower oesophageal sphincter relaxations on the basis that the nadir pressure at the gastro-oesophageal junction during swallow induced sphincter relaxation represented complete lower oesophageal sphincter relaxation. We believe that this strategy is valid.

The mechanism by which fundoplication interferes with the triggering of transient sphincter relaxations has yet to be defined but some data support the view that it is the result of changes to the distensibility of the gastric cardia because of the fundic wrap. The trigger zones for transient lower oesophageal sphincter relaxations have been evaluated in the dog by Franzi et al, ${ }^{14}$ who found that the most potent one was around the cardia, an area that would be buttressed by the fundoplication. Furthermore, in dogs, limiting distension of the cardia by banding, reduces the rate of belching and, by implication, the rate of transient lower oesophageal sphincter relaxations during gas insufflation. ${ }^{15}$

The other major effect of fundoplication was to prevent reflux from occurring during intervals of lower oesophageal sphincter relaxation. The proportion of transient sphincter relaxations accompanied by reflux fell from $47 \%$ to $17 \%$, and there was a five fold reduction in the number of reflux episodes occurring during swallow induced lower oesophageal sphincter relaxation. Precisely how fundoplication changes lower oesophageal sphincter function to achieve this effect is unclear but a likely factor is prevention of complete abolition of the high pressure zone at the gastro-oesophageal junction during sphincter relaxation. Our data support the findings of previous studies ${ }^{7111}$ that fundoplication renders swallow induced lower oesophageal sphincter relaxation incomplete. Our findings also show that fundoplication has a similar effect on transient lower oesophageal sphincter relaxations. Additionally, virtually no reflux episodes occurred because of absent basal sphincter pressure, or a drift in pressure to zero after surgery, whereas these mechanisms accounted for $15 \%$ of reflux episodes preoperatively.
How may fundoplication alter the pattern of lower oesophageal sphincter relaxation? It may lead to an alteration in the neural signals to the lower oesophageal sphincter so that the resultant neural inhibition is insufficient to give complete relaxation. this is, however, a less attractive hypothesis than the possibility that fundoplication renders lower oesophageal sphincter relaxation incomplete through a primary change in its mechanics. In dogs, fundoplication is associated with the presence of a sphincter like high pressure zone in the region of the wrap even after excision of the lower oesophageal sphincter. ${ }^{16}$ The most likely explanation for these observations and our own is that the fundic wrap itself exerts extrinsic compression in addition to lower oesophageal sphincter pressure. In patients who have undergone fundoplication, therefore, it may be more appropriate to talk of gastro-oesophageal junction pressure than lower oesophageal sphincter pressure.

Incomplete lower oesophageal sphincter relaxation may not be the only mechanism preventing reflux. About $50 \%$ of the transient sphincter relaxations that occurred postoperatively had nadir pressures within $2 \mathrm{~mm} \mathrm{Hg}$ of intragastric pressure, and thus were complete by preoperative criteria. The proportion of complete relaxations accompanied by reflux, however, fell substantially after fundoplication. The prevention of reflux during complete lower oesophageal sphincter relaxation suggests that there are other mechanical effects of fundoplication on sphincter function separate from that of simple extrinsic squeeze. In vitro studies suggest that the sphincteric length contributes to sphincter competence, ${ }^{17}$ and previous reports have suggested that fundoplication increases the length of sphincter exposed to intra-abdominal pressure. ${ }^{35}$ Postoperative studies, however, have not shown sphincter length to be important, either alone or in combination with basal lower oesophageal sphincter pressure, ${ }^{18}$ although an interaction between sphincter length and nadir sphincter pressure has not been examined. It must also be accepted that nadir pressures at the gastro-oesophageal junction during lower oesophageal sphincter relaxation are at the limits of resolution for manometry. Furthermore, undermeasurement by the sleeve sensor of the residual $1 \mathrm{mmHg}$ or so during lower oesophageal sphincter relaxation could have resulted in the under-recording of small but physiologically significant residual pressures during what seem to be complete lower oesophageal sphincter relaxations.

Three patients could be regarded as operative failures with recurrent pathological reflux, although only one was symptomatic. Nevertheless, even though acid exposure was still abnormal, fundoplication substantially reduced the number of reflux episodes but was associated with more prolonged acid clearance. The difference between the operative failure and success, therefore, seems to have been one of degree since fundoplication also reduced the number of transient lower oesophageal sphincter relaxations, albeit not to the same extent as in operative success. Importantly, however, the proportion 
of transient lower oesophageal sphincter relaxations associated with reflux was not reduced, although there were too few events for statistical comparison. The reason for this is unclear as basal and nadir lower oesophageal sphincter pressures were similar to those in patients in whom reflux was controlled. Could the antireflux effect of fundoplication be related to other factors not measured in the present study? This remains a possibility. Some workers have supported the concept that fundoplication produces a simple, one way mechanical flap or flutter valve. ${ }^{1119-21}$ Their observations, however, could also be explained on the basis of extrinsic compression of the lower oesophageal sphincter by the wrap.

Previously, investigations into the mechanism of action of fundoplication have been based on the hypothesis that effects on basal lower oesophageal sphincter pressure are relevant to the antireflux effect. This is an unappealing proposal in the light of what is now known about the mechanisms of gastro-oesophageal reflux. ${ }^{9}$ The hypothesis is not supported by the measurements themselves as neither postoeprative basal gastro-oesophageal junction pressure nor change in basal pressure after fundoplication correlated with the success or failure of surgery. ${ }^{67101819}$

In summary, we have investigated the antireflux mechanism of fundoplication by examining its effects on spontaneous patterns of reflux and associated oesophageal motility. Our data suggest that fundoplication exerts complex mechanical effects on the gastro-oesophageal junction, the principal consequences of which are to inhibit the triggering of transient lower oesophageal sphincter relaxations and to prevent the complete relaxation of the gastrooesophageal junction.

This study was supported by a grant from the Australian National Health and Medical Research Council. Dr Ireland was supported by a Travelling Fellowship from the Royal College of Physicians in Edinburgh. The results have been publish

abstract form, Gastroenterology, 1982;82:1042.
We gratefully acknowledge the assistance of Jill Hanley in the preparation of the manuscript.
1 Nissen R, Eine E, Einfat HE. Operation zur Beeinflussung der $\mathrm{R}$ defluxochitis Schwei\% Schreiz Med Wochenschr 1956;86: R deflux.

2 Richardson JD, Kuhns JG, Richardson RI, Polk HC. Properly conducted fundoplication reverses histologic evidence of esophagitis. Ann Surg 1983; 197: 763-70.

3 DeMeester TR, Johnson LF, Kent AH. Evaluation of current operations for the prevention of gastroesophageal reflux. operations for the prevention
Ann Surg 1974; 180: $511-25$.

4 DeMeester TR, Bonavina L, Albertucci M. Nissen fundoplication for gastroesophageal reflux disease. Evaluation of primary repair in 100 consecutive patients. Ann Surg 1986; 204: 9-20.

5 Johnsson F, Joelsson B, Gudmundsson K, Floren C-H, Walther B. Effects of fundoplication on the antireflux mechanism. BrF Surg 1987; 74: 1111-4.

6 Bierkeset T, Nordgard K, Schionsby H. Effect of Nissen fundoplication on the competence of the lower esophageal fundoplication on the competence of the lower

7 Gill RC, Bowes KL, Murphy PD, Kingma YJ. Esophageal motor abnormalities in gastroesophageal reflux and the motor abnormalities in gastroesophageal reflux and the

8 Dent J, Dodds WJ, Friedman RH, et al. Mechanism of gastroesophageal reflux in recumbent asymptomatic human subjects. F Clin Invest 1980; 65: 256-67.

9 Dent J, Holloway RH, Toouli J, Dodds WJ. Mechanisms of lower oesophageal sphincter incompetence in patients with symptomatic gastrooesophageal reflux. Gut 1988; 29: 1020-8.

10 Kiroff GK, Maddern GJ, Jamieson GG. A study of the factors responsible for the efficacy of fundoplication in the treatment of gastro-oesophageal reflux. Aust NZ7 Surg 1984; 54: 109-12.

11 Bowes KL, Sarna SK. Effect of fundoplication on the lower esophageal sphincter. Can f Surg 1975; 18: 328-32.

12 Dent J. A new technique for continuous sphincter pressure measurement. Gastroenterology 1976; 71: 263-7.

13 Holloway RH, Kocyan P, Dent J. Meals provoke gastroesophageal reflux by increasing the rate of transient lower oesophageal sphincter relaxations. Dig Dis Sci 1991; 36: 1034-9.

14 Franzi SJ, Martin CJ, Cox MR, Dent J. Response of canine lower oesophageal sphincter to gastric distension. $A m \mathcal{F}$

15 Strombeck DR, Griffen DW, Harrold D. Eructation of gas through the gastroesophageal sphincter before and after limiting distension of the gastric cardia or infusion of a Badrenergic amine in dogs. Am $\mathcal{I}$ Vet Res 1989; 50: 751-3.

16 Siewert R, Jennewein HM, Waldek F. Mechanism of action of fundoplication. Daniel EE, ed. Proceedings 4th International Symposium Gastrointestinal Motility. Vancouver: Mitchell Press, 1974: 145-52.

17 Pettersson GB, Bombeck CT, Nyhus LM. The lower esophageal sphincter: mechanisms of opening and closure. Surgery 1980; 88: 307-14

18 Bancewicz, Mughal M, Marples M. The lower oesophageal sphincter after floppy fundoplication. Br F Surg 1987; 74: $162-4$

19 Fisher RS, Malmud LS, Lobis IF, Maier WP. Antireflux surgery for symptomatic gastroesophageal reflux: mechsurgery for symptomatic gastroesophageal refl
anisms of action. Dig Dis $S$ ci 1978; 29: 152-60.

20 Butterfield WC. Current hiatal hernia repairs. Similarities, mechanisms and extended indications: an autopsy study. mechanisms and extended

21 Matikainen M, Kaukinen L. The mechanisms of action of Nissen fundoplication. Acta Chir Scand 1984; 150: 653-5. 